\title{
Retortamonas intestinalis
}

National Cancer Institute

\section{Source}

National Cancer Institute. Retortamonas intestinalis. NCI Thesaurus. Code C124369.

A species of Retortamonas protozoa that form cysts and trophozoites in the large

intestines of humans. Infestation with R. intestinalis occurs through ingestion of cysts in

fecal-contaminated food or water. The species is considered non-pathogenic. 